\title{
Estudo documental das políticas de prevenção e promoção em saúde no Haiti
}

\author{
Jean Bart David
}

\author{
Jean Bart David \\ Membro do grupo de pesquisa de Políticas de Saúde da Universidade \\ Estadual do Oeste do Paraná. \\ Email: jeanbartdvd326@gmail.com \\ ORCID: https://orcid.org/0000-0001-5031-0363
}

\begin{abstract}
Resumo
Principais causas de morbimortalidade na população haitiana são as doenças infecciosas e as doenças do sistema circulatório. Segundo a Organização Pan-americana de Saúde, as principais causas de mortalidade no país nos últimos anos foram as doenças infecciosas representando $14 \%$ das mortes e as doenças do sistema circulatório $20 \%$. Considerando os aspectos acima relacionados acredita-se que uma maior compreensão sobre as políticas de prevenção e promoção em saúde aplicado pelo estado do Haiti permitirá compreender se essas políticas atendem as verdadeiras demandas em saúde da população haitiana. Ante exposto, define-se o objetivo da pesquisa como: Analisar as políticas de prevenção e promoção em saúde do estado do Haiti através dos documentos publicados no site oficial do Ministério da Saúde e a População. Adotou-se a abordagem qualitativa, através de uma pesquisa documental utilizando fontes secundários sobre as políticas em promoção em saúde do Estado da República do Haiti. A análise de conteúdo proposta por Minayo foi usada como forma de análise dos documentos selecionados. Foram analisados sete documentos, constatou-se que as políticas de promoção em saúde do Estado do Haiti têm foco maior na prevenção e reparação de danos à saúde. A constituição do Haiti de 1987 aborda a questão de saúde, mas sem referência à questão da promoção em saúde.
\end{abstract}

\section{Palavras-Chave}

Promoção da saúde, Política Pública, Haiti

\begin{abstract}
The main causes of morbidity and mortality in the Haitian population are infectious diseases and diseases of the circulatory system. Considering the above aspects, it is believed that a greater understanding of the prevention and health promotion policies applied by the state of Haiti will allow us to understand if these policies meet the true health demands of the Haitian population. Given the above, the research objective is defined as: To analyze the health prevention and promotion policies of the state of Haiti through the documents published on the official website of the Ministry of Health and Population. The qualitative approach was adopted through documentary research using secondary sources on health promotion policies of the State of the Republic of Haiti. The content analysis proposed by Minayo was used as a way of analyzing the selected documents. Seven documents were analyzed. It was found that the health promotion policies of the State of Haiti have a greater
\end{abstract}


Jean Bart David

focus on the prevention and repair of health damage. Haiti's 1987 constitution addresses the issue of health, but without reference to the issue of health promotion.

\section{Keywords}

Health Promotion, Public policy, Haiti

\section{Introdução}

No ano de 2010, um terremoto de magnitude aproximadamente 7 atingiu o Haiti enfraquecendo ainda mais as estruturas sanitárias no país. Na última década, a taxa de mortalidade materna relatada no país foi estimada em 157 mortes por 100.000 nascidos vivos e apenas $43 \%$ das unidades de saúde ofereciam serviços de parto (ORGANIZAÇÃO PAN-AMERICANA DE SAÚDE ET AL., 2019) . Outro aspecto que pode ser considerado como um problema de saúde pública na última década, é o aumento das taxas de incidências de homicídios e abusos físicos sofridos por parte das mulheres. A taxa de homicídios foi estimada em 10,2 mortes por 100.000 habitantes e mais de um quarto das mulheres de 15 a 49 anos (28\%) relataram ter sofrido abuso físico aos 15 anos e $13 \%$ das mulheres nessa faixa etária relataram ter sofrido abuso sexual aos 15 anos de idade em algum momento da sua vida (ORGANIZAÇÃO PAN-AMERICANA DE SAÚDE ET AL., 2019).

As principais causas de morbimortalidade na população haitiana são as doenças infecciosas, as doenças do sistema circulatório. As principais causas de mortalidade no país nos últimos anos foram as doenças infecciosas representando $14 \%$ das mortes e as doenças do sistema circulatório 20\% (ORGANIZAÇÃO PAN-AMERICANA DE SAÚDE ET AL., 2019). Outro fator que mais causas mortes no país nos últimos anos foram os sinais e sintomas não classificados. A taxa de morte pelos sinais e sintomas não classificados foram de $32 \%$ nos últimos anos e a esperança de vida é de 63, 33 anos(ORGANIZAÇÃO PAN-AMERICANA DE SAÚDE ET AL., 2019).

. Várias organizações internacionais se fazem presentes no Haiti e atuam principalmente no setor de saúde. Considerando o quadro de morbimortalidade da população haitiana, os diferentes programas de promoção em saúde não atendem as verdadeiras demandas em saúde, deixando de lado as doenças que mais causam mortes nas últimas décadas no Haiti. A promoção da saúde permite que as pessoas melhorem sua própria saúde, abrange uma ampla gama de intervenções sociais e ambientais destinadas a promover e proteger a saúde individual e a qualidade de vida, abordando as principais causas de doenças, incluindo a prevenção, e não apenas enfocando no tratamento e cura (ORGANIZAÇÃO MUNDIAL DA SAÚDE, 2019). No contexto da prevenção em saúde, definiuse como um conjunto de medidas sanitárias, técnicas e educacionais destinadas a prevenir o aparecimento de um problema de saúde, sua transformação em uma doença declarada ou sua recorrência ou suas complicações.

Os conceitos de prevenção e promoção em saúde tem suas distinções, a prevenção centra-se na doença e risco e refere-se a uma concepção particular de saúde como ausência de doença, enquanto a promoção da saúde projeta essas estratégias de maneira sinérgica, com base em uma abordagem intersetorial. Considerando esses aspectos acima relacionados acredita-se que uma maior compreensão sobre as políticas de prevenção e promoção em saúde aplicado pelo estado do Haiti permitirá compreender se essas políticas atendem as verdadeiras demandas em saúde da população haitiana. Ante exposto, define-se com problema desta pesquisa: qual o rumo das políticas de promoção em saúde no Haiti? 
Para alcançar os objetivos, adotou-se a abordagem qualitativa baseando numa pesquisa documental utilizando fontes secundários sobre políticas de promoção em saúde do Estado do Haiti. Os resultados demonstram que a política nacional de promoção em saúde no Haiti ainda não está implementada. Diversos problemas de ordem organizacionais e econômicas que enfrenta o país, impede que as ações em voltadas para promoção em saúde ainda são poucas, por outro lado, as ações de prevenção de danos em saúde foram mais destacadas. Foram analisados sete documentos, constatou-se que as políticas de promoção em saúde do Estado do Haiti têm foco maior na prevenção e reparação de danos à saúde. $A$ constituição do Haiti de 1987 aborda a questão de saúde, mas sem referência à questão da promoção em saúde.

Metodologia

\begin{tabular}{|c|c|c|c|}
\hline Instrumentos & $\begin{array}{l}\text { Ano de } \\
\text { publicação }\end{array}$ & Objetivos & Promoção em Saúde \\
\hline $\begin{array}{l}\text { Constitution de la } \\
\text { République d'Haïti }\end{array}$ & 1987 & $\begin{array}{l}\text { Garantir direitos } \\
\text { inalienáveis e } \\
\text { imprescritíveis à vida, à } \\
\text { liberdade e à busca da } \\
\text { felicidade }\end{array}$ & $\begin{array}{l}\text { Art.19- direito à vida e a } \\
\text { saúde } \\
\text { Art.23-restaurar a saúde } \\
\text { através da criação de } \\
\text { hospitais, centros de saúde } \\
\text { e clínicas. } \\
\text { Art 22: O Estado reconhece } \\
\text { o direito de todo cidadão a } \\
\text { moradia digna, educação, } \\
\text { alimentação e previdência } \\
\text { social. }\end{array}$ \\
\hline $\begin{array}{l}\text { Politique Nationale de } \\
\text { Santé dans la } \\
\text { republique D'Haïti }\end{array}$ & 2012 & $\begin{array}{l}\text { Garantir a redução da } \\
\text { morbimortalidade, } \\
\text { relacionada aos principais } \\
\text { problemas de saúde } \\
\text { identificados }\end{array}$ & $\begin{array}{l}\text { Proteção em Saúde } \\
\text { - Garantia de acesso } \\
\text { equitativo e universal aos } \\
\text { cuidados de saúde } \\
\text { qualidade centrada } \\
\text { na pessoa }\end{array}$ \\
\hline $\begin{array}{l}\text { Politique nationale de } \\
\text { promotion de la Santé }\end{array}$ & 2011 & $\begin{array}{l}\text { Define a política nacional } \\
\text { de promoção em saúde }\end{array}$ & $\begin{array}{l}\text { Promoção em saúde } \\
\text { - Janeiro de 2006: criação } \\
\text { da Direção de Promoção da } \\
\text { Saúde e Proteção } \\
\text { Ambiental (DPSPE). } \\
\text {-Adoção da carta de }\end{array}$ \\
\hline
\end{tabular}


Jean Bart David

\begin{tabular}{|c|c|c|c|}
\hline & & & $\begin{array}{l}\text { promoção à saúde } \\
\text { considerando a política } \\
\text { nacional de saúde } \\
\text {-Plano estratégico: } \\
\text { promoção da saúde como } \\
\text { um dos programas } \\
\text { prioritários do MSPP }\end{array}$ \\
\hline $\begin{array}{l}\text { Plan strategique } \\
\text { national pour la } \\
\text { prevention et le } \\
\text { contrôle des IST et du } \\
\text { VIH/sida en Haiti }\end{array}$ & 2002 & $\begin{array}{l}\text { Análise da situação das } \\
\text { doenças sexualmente } \\
\text { transmissíveis (VIH) }\end{array}$ & $\begin{array}{l}\text { Promoção e Prevenção em } \\
\text { saúde } \\
\text {-Promover um } \\
\text { comportamento sexual } \\
\text { mais seguro e reduzir a } \\
\text { vulnerabilidade de grupos } \\
\text { específicos } \\
\text {-Promoção de } \\
\text { comportamento sexual } \\
\text { seguro, promoção / } \\
\text { distribuição e venda de } \\
\text { preservativos, redução da } \\
\text { transmissão cruzada de } \\
\text { mãe para filho e segurança } \\
\text { da transfusão de sangue. }\end{array}$ \\
\hline $\begin{array}{l}\text { Plan stratégique } \\
\text { national de santé } \\
\text { sexuelle et } \\
\text { reproductive }\end{array}$ & 2018 & $\begin{array}{l}\text { Contribuir para reduzir o } \\
\text { número de gravidez } \\
\text { precoces e / ou } \\
\text { indesejadas e o número } \\
\text { de infecções por DST / } \\
\text { HIV entre jovens de } 15 \text { a } \\
24 \text { anos }\end{array}$ & $\begin{array}{l}\text { Promoção da saúde } \\
\text {-Mobilizar a sociedade civil, } \\
\text { autoridades locais eleitas, } \\
\text { em torno de um plano } \\
\text { eficaz para promover a } \\
\text { saúde sexual e reprodutiva } \\
\text { e os direitos das mulheres } \\
\text { e meninas } \\
\text { Prevenção em saúde } \\
\text {-Fortalecer o conhecimento } \\
\text { de jovens de } 10 \text { a } 24 \text { anos } \\
\text { sobre a prevenção da } \\
\text { sexualidade e os direitos } \\
\text { dos jovens nas escolas e } \\
\text { mídias sociais. }\end{array}$ \\
\hline
\end{tabular}


Jean Bart David

\begin{tabular}{|l|l|l|l|}
\hline & & & \\
\hline $\begin{array}{l}\text { Projet de renforcement } \\
\text { de la santé primaire et } \\
\text { de la surveillance } \\
\text { épidémiologique en } \\
\text { Haïti }\end{array}$ & 2018 & $\begin{array}{l}\text { Fortalecimento da } \\
\text { prestação de serviços de } \\
\text { atençáo primária à saúde. }\end{array}$ & $\begin{array}{l}\text { Prevenção em saúde } \\
\text {-Prevenção de doenças } \\
\text { infecciosas e a melhor } \\
\text { gestão de resíduos médicos } \\
\text { éáguas residuais }\end{array}$ \\
\hline $\begin{array}{l}\text { Fortalecimento da } \\
\text { vigilância e controle de } \\
\text { doenças infecciosas } \\
\text { Slan Stratégique du } \\
\text { pour la Réforme }\end{array}$ & 2005 & $\begin{array}{l}\text { 240 } \\
\text { Tornar os cuidados } \\
\text { primários de saúde } \\
\text { acessíveis à população. }\end{array}$ & $\begin{array}{l}\text { Promoção da saúde } \\
\text { fortalecer o quadro de } \\
\text { intervenção no campo da } \\
\text { educação da população no } \\
\text { domínio da saúde e da } \\
\text { proteção do ambiente }\end{array}$ \\
\hline
\end{tabular}

Este estudo adotou a abordagem qualitativa, através de uma pesquisa documental utilizando fontes secundários sobre as políticas em promoção em saúde do Estado da República do Haiti. "[...] a pesquisa documental vale-se de materiais que não receberam ainda um tratamento analítico, ou que ainda podem ser reelaborados de acordo com os objetivos da pesquisa" (GIL P. 70, 2008).

Os documentos foram selecionados no site http://mspp.gouv.ht e http://www.omrh.gouv.ht. Para seleção dos documentos, foi considerando a temática "Prevenção e promoção em saúde". A análise de conteúdo proposta por Minayo foi usada como forma de análise dos documentos selecionados (MINAYO, 2014). Para a fase de Préanálise, escolheu-se os documentos a serem analisados a partir de busca através do site governamentais do estado do Haiti. Na fase de Exploração do material, objetivou-se realizar uma classificação dos textos selecionados considerando as temáticas principais: promoção em saúde e prevenção de saúde. Na última fase, Tratamentos dos resultados obtidos e interpretação dos documentos estudados, foi feito a organização dos documentos em um quadro referente ao contexto das políticas de promoção em saúde no Estado do Haiti.

\section{Resultados e Discussões}

Foram analisados sete documentos, constatou-se que as políticas de promoção em saúde do Estado do Haiti têm foco maior na prevenção e reparação de danos à saúde. A constituição do Haiti de 1987 aborda a questão de saúde, mas sem referência à questão da promoção em saúde. As principais ações em promoção em saúde foram a criação da direção de promoção da saúde e proteção ambiental (DPSPE), adoção da carta de promoção à saúde e o desenvolvimento de um plano estratégico nacional. Diversos problemas de ordem organizacionais e econômicos impedem a implementação e direcionamento da promoção em 
Jean Bart David

saúde no país. Segundo o Ministério da saúde e da População do Haiti, o problema de ordem econômica que enfrenta o país, impede a implementação de políticas de saúde para diminuir as doenças com maior taxa de incidência no país. Por outro lado, depois de terremoto de 2010, o número de organizações internacionais que atuam no setor de saúde vem aumentando nos últimos anos (HAITI, 2018), mas suas ações não têm impacto significante na redução das doenças com maior taxa de morbimortalidade no país. No quadro 1 , são apresentados os principais programas do governo do estado do Estado que tem como temática: prevenção e promoção em saúde. No quadro 1, são apresentados os principais programas do governo do estado do Estado que tem como temática a temática: promoção em saúde.

\section{Quando 1- Programas de políticas de promoção em saúde do Estado do Haiti}

Fonte: dados elaborados pelo autor a partir dos documentos selecionados nos sites: http://mspp.gouv.ht e http://www.omrh.gouv.ht.

\section{Prevenção e Promoção em Saúde no Haiti}

O quadro das doenças com maior incidência de morbimortalidade no Haiti nas últimas décadas são as doenças infeccionas. Depois do terremoto, a incidência de mortes causadas pela cólera, doença trazida pelos soldados da missão de paz das organizações das nações unidas (MINUSTAH) teve uma incidência alta. Segundo o Ministério da Saúde e da População, nos primeiros meses do surgimento da cólera no Haiti, teve aproximadamente 3.166 casos semanais em média, e o quinto pico ocorreu em novembro de 2013 com 2.811 casos por semana (MINISTÉRIO DA SAÚDE E DA POPULAÇÃO DO HAITI, 2018). Considerando o quadro das causas de morbimortalidade no Haiti, nos documentos analisados, o direcionamento das ações de prevenção e promoção em saúde não atende as demandas em saúde da população.

Nesse contexto, a falta de atendimento das verdadeiras demandas em saúde da população pode ter impacto grave no estado de saúde da população em geral. O estado de saúde é caracterizado por interações entre fatores socioeconômicos e interdependentes com o ambiente físico, comportamento individual e não agem isoladamente: é a combinação de seus efeitos que influenciam o estado de saúde. A prevenção em saúde é o conjunto de medidas destinadas a evitar ou reduzir o número e gravidade de doenças ou acidentes (ARWIDSON ET AL., 2017). Nesse sentido, considerando o direcionamento das políticas de prevenção e promoção em saúde do Haiti, as ações em saúde são direcionadas para prevenção de danos em saúde.

Por outro lado, ações voltadas para promoção em saúde são escassas e a política de promoção em saúde no país ainda não está implementada considerando os diversos problemas enfrentados pelo sistema pública de saúde haitiana. Os benefícios das ações de promoção em saúde para a população que vive em condições precárias gerariam grandes benefícios para sua saúde. Os indivíduos e grupos podem ganhar o controle de suas vidas e adquirir maior controle sobre as decisões e ações que afetam sua saúde no contexto de mudar sua ambiente social. Destaca- se que a promoção em saúde tem relação com a autonomia e o empoderamento dos indivíduos na participação de melhoria da sua saúde (CARVALHO ET AL; 2017). 
Jean Bart David

Ao analisar o documento da política nacional de promoção em saúde no Haiti, apesar que essa política segue o direcionamento do conceito de promoção em saúde GLOBAL (BRASIL, 2002), por outro lado, os diversos problemas enfrentados pelo sistema de saúde impedem a implementação dessa política na pratica.

Considerando esses aspectos, as políticas de prevenção e promoção em saúde no Haiti ainda são muito precárias, o país sofre com instabilidade política e econômica que impede a restauração dos serviços sanitários. A promulgação da Constituição de 1987 e a opção do povo haitiano por regimes políticos do tipo democrático forneceram esperança. No entanto, a falta de consenso por parte dos vários setores políticos para um projeto social e a incapacidade dos diferentes atores em responder às exigências políticas e organizacionais de uma nova ordem democrática causaram vários problemas nos diferentes setores (publicas e privadas). A falta de implementação de políticas setoriais explícitas e políticas transversais que colocam o país no caminho para o desenvolvimento sustentável, privou o país até agora $\mathbf{2 4 2}$ do foco necessário para desenvolver planos conjuntos e programas eficazes para a população (Jean 1976).

\section{Considerações finais}

Nos documentos analisados, constatou-se os diferentes problemas de saúde da população haitiana não foram considerados ao implementar políticas de promoção em saúde no país. O sistema de saúde pública do país enfrenta vários problemas como: organizativos, financiamento, recursos humanos, entre outros. Considerando tais aspectos, as diferentes ações em saúde devem ser bem planejadas no sentido de focar esforços nas verdadeiras problemas de saúde da população haitiana. O compromisso político com a saúde requer estratégias abrangentes envolvendo todos os setores da sociedade, nesse aspecto, trata-se de dar às pessoas as habilidades para melhorar sua própria saúde e a da comunidade em que vivem.

Considerando os vários desafios do sistema público de saúde haitiano, o setor da saúde deve fazer alianças com as diversas entidades da sociedade, em particular a mídia, a academia, os profissionais e o público em geral. Da mesma forma, é a responsabilidade da população em geral em estabelecer as modalidades de defesa da saúde, levando em consideração o clima político, econômico e social.

(Recebido para publicação em agosto de 2019)

(Reapresentado em novembro de 2019)

(Aprovado para publicação em dezembro de 2019)

\section{Cite este artigo}

DAVID, Jean, 2019. Estudo documental das políticas de prevenção e promoção em saúde no Haiti. Revista Estudos Políticos: a publicação semestral do Laboratório de Estudos Hum(e)anos (UFF).Rio de Janeiro, Vol.10|N.2, pp.236-244, dezembro de 2019. 
Jean Bart David

\section{Referências bibliográficas}

ORGANISATION PANAMÉRICAINE DE LA SANTÉ, ORGANISATION MONDIALE POUR LA SANTÉ. SANTÉ DANS LES AMÉRIQUE. Disponivel em: https://www.paho.org/salud-en-las-americas-

2017/?page_t_fr=haiti\&lang=fr. Acessado em: 29 de jun. de 2019.

ORGANIZAÇÃO PAN-AMERICANA DA SAÚDE, OMS. Organizacíon Panamericana de la salud. Disponível em: https://www.paho.org/hq/index.php?lang=es. Acessado em: 12 de jun. de 2019

ORGANIZAÇÃO MUNDIAL DA SAÚDE. Organisation 243 Mondiale de Santé. Disponivel em: https://www.who.int/features/qa/health-promotion/fr/. Acessado em: 27 de jun. de 2019.

ARWIDSON PIERRE, BURY JAQUES, CHOQUET MARIE, PERETTI CRISTINE DE, PAICHELER GENENIÉR, ET AL. Education pour la santé des jeunes: démarches et méthodes. 2001st ed. Paris, France: Les éditions Inserm; 2017. 269 p.

GIL ,AC. Métodos e ténicas da pesquisa social [Internet]. 6th ed. Editora Atlas S.A, editor. Vol. 29, Educação e Pesquisa. São Paulo: Editora Atlas S.A; 2008. 1-201 p.

MINAYO, Maria C.S. O desafio do conhecimento: Pesquisa Qualitativa em Saúde. 14th ed. Hucitec, editor. São Paulo; 2014. 407 p.

MINISTÈRE DE LA SANTÉ PUBLIQUE ET DE LA POPULATION. 2005;(Plan Stratégique National pour la Réforme du Secteur de la Santé). Disponivel em: http://mspp.gouv.ht/newsite/. Acessado em: 29 de jun. de 2019

RÉPUBLIQUE D 'HAITI,. MINISTRE DE LA PLANIFICATION ET DE LA COOPÉRATION EXTERNE. Dispomivel em: http://www.sgcm.gouv.ht/. Acessado em: 28 de jun. de 2019

RÉPUBLIQUE D'HAITI. MINISTÉRE DE LA SANTÉ PUBLIQUE ET DE LA POPULATION. Disponivel em: http://mspp.gouv.ht/newsite/. Acessado em: 29 de jun. de 2019

CARVALHO, COHEN , AKERMAN . Refletindo sobre o instituído na Promoção da Saúde para problematizar "dogmas." Saúde em Debate. 2017;41(spe3):265-76.

CARVALHO . Os múltiplos sentidos Empowerment.pdf. 2004;20(4):1088-95. 
Jean Bart David

BRASIL. MINISTÉRIO DA SAÚDE. SECRETARIA DE POLÍTICAS DE SAÚDE. AS cartas da promoção da saúde. IV. Saúde M da, editor. Vol. 52, / Ministério da Saúde, Secretaria de Políticas de Saúde. Brasilia; 2002. 1-5 p.

JEAN . Structures économiques et lutte nationale populaire en Haiti. Haiti: Editions Nouvelle Optique; 1976. 